\title{
EDITORIAL
}

\section{EFFETS DES RESERVES D'EAU EN AMONT DES USINES DE TRAITEMENT D'EAU POTABLE}

Dans ce type de problème, il est très difficile de généraliser sous peine de conclusions hâtives qui peuvent être partiellement fausses.

II existe divers types de retenues, les buts étant différents, ainsi que les temps de séjour de l'eau, les profondeurs et bien sûr les avantages et les inconvénients qui leur sont associés.

Tout d'abord le but : certaines retenues ne sont utilisées pour l'alimentation de l'usine d'eau potable que secondairement. Ce sont des barrages, des retenues, leur rôle premier étant soit la régulation des étiages, soit l'écrètement de crues. Elles peuvent être aussi utilisées pour la production d'électricité : elles ont en commun d'avoir des temps de séjour de l'ordre de l'année, et des profondeurs supérieures à $10 \mathrm{~m}$.

D'autres retenues ont été construites volontairement par le traiteur d'eau afin d'avoir soit une sécurité en cas de pollution (réserve d'eau, ou retardement de l'arrivée du polluant) soit pour un effet d'écrètement des pointes de pollution (effet tampon). Là, il faudra bien distinguer ces 2 buts car la construction de la réserve sera différente.

Ce type de retenue a en commun, d'avoir des temps de séjour de l'ordre de la semaine et même parfois moins de 2 à 3 jours, d'avoir des profondeurs qui n'atteignent qu'exceptionnellement les $10 \mathrm{~m}$. D'après ce classement assez sommaire en deux groupes, nous pouvons d'ores et déjà voir que les problèmes et les avantages associés à chacune de ces catégories seront différents.

En ce qui concerne les retenues ayant des temps de séjour de l'ordre de 1 an, le principal avantage, est d'avoir la quantité d'eau nécessaire à l'usine quelle que soit la saison et notamment l'été où les petites rivières les alimentant sont quasiment à sec.

C'est la principale raison du choix du site.

Par contre les inconvénients sont indéniables, et le traiteur d'eau doit les prendre en compte lors de la conception de l'usine :

- Problèmes d'algues : il faudra prévoir des prises d'eau à des hauteurs différentes.

- Problèmes de stratification. En général on constate une stratification de Mai à Novembre. Cette stratification entraîne dans le fond de la retenue, l'absence totale d'oxygène, la présence de fer, de manganèse, de carbone organique, de composés sapides. 
Comme ces réserves d'eau, bien souvent sont utilisées pour réguler les étiages, en été, il est impossible de neprendre autre chose que cette eau de fond.

Les usines utilisant de telles eaux demandent à être très sophistiquées, d'autant plus que l'eau est peu minéralisée ; sans effet tampon, car à ces problèmss s'ajoutent les changements très importants de $\mathrm{pH}$ entre le jour et la nuit. (Fonction chlorophylliènne des algues).

En général il faut:

Une aération ; une stabilisation du $\mathrm{pH}$, un traitement au permanganate de potassium, une coagulation avec un sel d'aluminium, une filtration sur sable, une deuxième correction de $\mathrm{pH}$, une ozonation et une filtration sur charbon actif. Toutes ces étapes étant absolument nécessaires. De plus, il faut que l'usine soit régulée automatiquement pour tenir compte des variations journalières importantes.

En ce qui concerne les réserves d'eau (bassin de stockage) en amont des prises d'eau avec des temps de séjour de l'ordre de la semaine: Dans ce cas elles sont conçues par le traiteur d'eau et doivent être construites en fonction de l'usine et des besoins. C'est tout le contraire de ce qui a été traité au paragraphe précédent où l'usine devait s'adapter à la qualité de l'eau de la réserve.

Cette prise en compte du problème devait suivre cette voie logique, ce n'est pas toujours le cas et bien souvent le bassin peut être à l'origine d'une dégradation de l'eau.

Pour bien optimiser la réserve d'eau, il faut bien définir son objectif. C'est une mauvaise définition de l'objectif qui peut être à l'origine de désagréments.

Une telie réserve peut être nécessaire pour trois raisons. Ces trois raisons pouvant être ou ne pas être indépendantes.

a) L'usine a éliminé le traitement de l'ammoniaque par chloration et l'a remplacée par une oxydation biologique dans les décanteurs et les filtres, avec un rattrapage éventuel en fin de traitement par une chloration finale.

Dans ce type de traitement, il faut un effet tampon, afin d'éliminer toute variation brusque de la teneur en ions $\mathrm{NH}_{4}^{+}$dans l'eau brute.

De même, on peut avoir au cours de la journée des pointes de pollutions très brèves, rendant l'eau impropre à faire de l'eau potable selon la directive des C.E.E. en 1975.

Dans ce cas, il est impératif de mélanger l'eau très rapidement et de diluer les pointes de pollution. Le bassin sera donc conçu comme un réacteur à mélange le plus parfait possible en jouant sur la forme du bassin, la vitesse d'injection de l'eau, l'angle d'injection de l'eau.

Par contre si une pollution massive arrive sur la rivière ; toute l'eau de la réserve sera polluée et dans certains cas devra être évacuée.

b) L'usine est en aval immédiat d'une source de pollution et n'a pas assez de délai de réponse pour éviter une pollution accidentelle. Dans ce cas la réserve aura pour but «d'ajouter fictivement» quelques kilomètres de rivière afin de donner un temps de réponse supplémentaire pour soit arrêter ou ralentir l'usine, soit augmenter le taux de traitement.

Dans ce cas, il ne faudra surtout pas mélanger l'eau avec celle de la réserve comme dans le paragraphe précédent. On installera un système de chicanes permet- 
tant d'avoir d'une part, un temps de réponse plus long et d'autre part une circulation en piston afin de pouvoir localiser avec précision la partie polluée, pour éventuellement ne pas la laisser entrée sur l'usine de trai tement elle-même.

c) La troisième utilisation de cette réserve peut être d'avoir une masse d'eau pouvant permettre un arrêt d'entrée d'eau de la rivière sans pour autant arrêter l'usine.

Dans ce cas, le niveau dans la réserve sera changé et abaissé. On devra dans ce cas tenir compte de l'interaction des sédiments.

Ces bassins quelqu'ils soient sont des décanteurs et peu à peu se remplissent de sédiments. Si le fond du bassin est en relation avec la nappe phréatique, il y aura un équilibre de pression et tant que le sens de l'eau sera du bassin vers la nappe, seule la qualité de l'eau de la nappe sera affectée par l'eau de percolation à travers les sédiments. Si on abaisse le niveau de la réserve d'eau et que l'eau de la nappe alimente le bassin, il y aura risque de pollutions par l'eau de percolation à travers les sédiments. Cela diminuera d'un facteur très important la réserve effective d'eau.

Dans ce cas, il faudra d'une part rendre ce bassin étanche et éviter toute percolation de l'eau de la nappe à travers les sédiments et d'autre part éliminer régulièrement la masse de sédiments qui s'accumule.

Dans ces bassins à temps de séjour de l'ordre de la semaine, le problème de stratification n'existe pas, les problèmes d'algues sont négligeables. Le principal problème est l'effet de court circuit qui immobilise une partie de l'eau, dans ce cas alors les problèmes d'algues arrivent. II est donc impératif de bien concevoir ces bassins.

Dans le cas d'une usine qui a à satisfaire les 3 besoins, il faut tout d'abord des chicanes pour permettre un délai de réponse avec à l'entrée dans le bassin une station d'alerte automatique. Ensuite un bassin tampon servant de réserve d'eau (étanche et à circulation rapide).

Dans ces conditions toutes les sécurités sont réunies.

Dans la série de conférences qui est présentée vous pourrez voir tous ces types de bassins, ainsi que les inconvénients inhérents à l'utilisation d'un bassin déjà existant, non adapté aux besoins réels de l'usine.

Et je laisse le lecteur seul juge de la bonne ou mauvaise conception de réserves qui nous seront décrites.

A. MONTIEL 